\title{
Cognitive style differences in signal detection/discrimination
}

Alexey N. Gusev, Nataliia N. Volkova, Lomonosov Moscow State University

* The role of the factors, related to the manifestation of individual differences, is severely underestimated in psychophysics. In that regard, we highlight the necessity of taking into consideration both stimulation and individual differences factors.

* Therefore we carried out an experiment, in which task type and difficulty level were considered as stimulation factors, and cognitive styles (CS) were considered as individual differences factors.

* Thus, we revealed individual differences in sensory sensitivity index $A^{\prime}$, driven by cognitive style characteristics on the one hand, and task characteristics on the other hand.

* 98 participants with normal or correctedto-normal vision.

* IBM-compatible PC with a clean Windows XP Professional 32 bit operating system, in which all background processes were turned off.

* 22" LCD monitor, with a resolution of 1920 $\times 1080$. Participants viewed the monitor from a distance of $60 \mathrm{~cm}$

* Since our tasks suggest short duration of stimulus presentation, the latter was administered through retrace control procedure.

* RT was registered using a special USB response pad, providing the precision of \pm $5 \mathrm{~ms}$.

* Generalized linear models procedure with LSD multiple comparisons test using IBM SPSS Statistics 22
Stimuli and task

Visual signal detection task ('yes-no'):

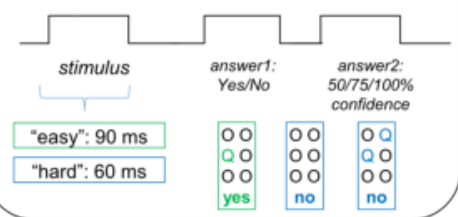

Audial signal discrimination task ('same-different'):

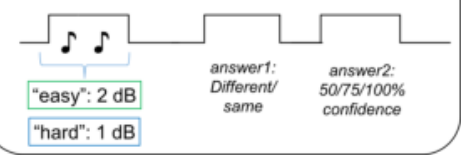

\section{Flexibility-Rigidity}

Refers to the extent of differences in reactions to stimulus fileds containing contradictory cues.

\section{Color-Word Test.}

\section{RED BLUE GREEN RED BLUE YELLOW}

\section{Focusing-Scanning}

Refers to individual consistency in attention allocation strategies.

Focusers differ from scanners in the amount of information sampled before commitment to a response.

Size Estimation Test.

All experiment tasks were created using

'Practice MSU' integrated computer system (http://psychosoft.ru).
We found significant differences in sensory sensitivity index $A^{\prime}$ between 'flexible' and 'rigid' subjects in both easy $(p=0.009)$ and hard $(p=0.004)$ Yes-No tasks.

* 'Scanners' showed significantly highe sensitivity in both easy $(p=0.044)$ and hard $(p=0.044)$ Yes-No tasks.

* The results indicated that 'flexible' subjects and 'scanners' showed higher sensitivity performing Yes-No task compared to 'rigid' and 'focusers', respectively. Moreover, 'flexible scanners' were revealed as the most successfu group compared to others.

* Significant between-group differences were only found in Yes-No tasks performance, but not in Same-Different tasks one due to the difference in task conditions.

Figure 1. Mean A' indices in easy (circle, solid line) and hard (square, dashed line) yes-no tasks
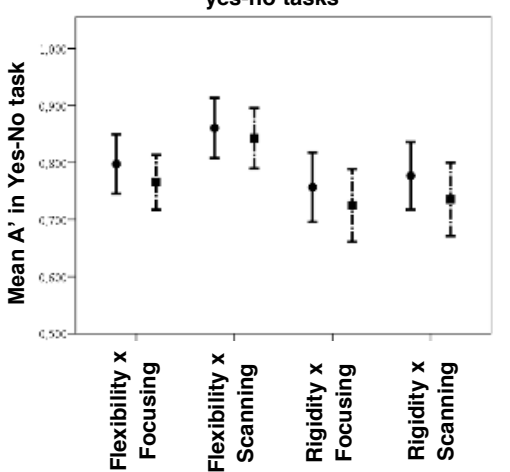

* The procedure of Yes-No task implies the necessity to inhibit automatic impulsive response 'yes' to 'distractor' - the stimulus with two target letters instead of one. Since both 'flexibility' and 'scanning' CS dimensions refer to the inhibition of automatic reactions to irrelevant stimulation, correspondent groups showed advantage in accuracy of solving this task.

* Same-Different task, in its turn, does not require the incorporation of such CS resources, and therefore both groups reached out to the same level of task performance.

Figure 2. Mean A' indices in easy (circle, solid line) and hard (square, dashed line) same-different tasks

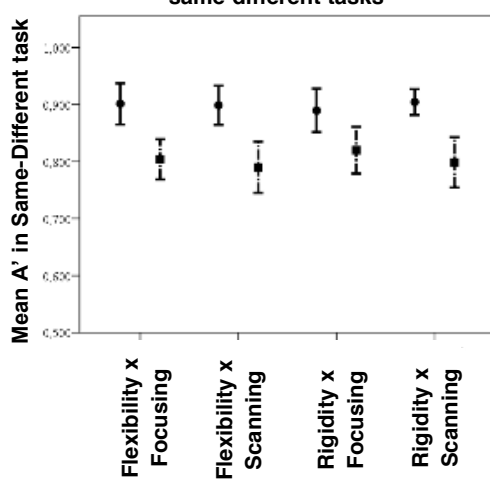

